\title{
Bioinformatics Analysis of Metabolomics Data Unveils Association of Metabolic Signatures with Methylation in Breast Cancer
}

\author{
Fadhl M. Alakwaa, ${ }^{*, \dagger}$ Masha G. Savelieff ${ }^{\dagger}$
}

†Department of Neurology, University of Michigan, Ann Arbor, MI 48109, USA *Corresponding author email: alakwaaf@med.umich.edu

Table of contents:

The following supporting information is available free of charge at ACS website http://pubs.acs.org. The supporting information contains the analyses to determine ideal $k$ ( $k$-means and pam for dataset 1 ), pam analysis for $k=4$ and 3 of dataset 1 , clustering of dataset 2 , SAM of dataset 2 , and association between $\mathrm{k}$-means clusters of dataset 2 and patients' molecular subtypes.

Figure $\mathbf{S} 1$. $\mathbf{k}$ value determination for $\mathbf{k}$-means. Analysis to find optimal $\mathrm{k}$ value for k-means clustering of dataset 1 by Elbow, Silhouette methods, and Gap statistic.

Figure S2. Unsupervised pam clustering defines distinct metabolic subtypes of BC tumors. pam (partition around mediods) of dataset 1 identified metabolomics clusters using both, (A) $k=4$ (current number of receptor subtypes) and (B) k=3 (ideal k value by Elbow method and Gap statistic).

Figure S3. $\mathbf{k}$ value determination for pam. Analysis to find optimal $k$ value for pam clustering of dataset 1 by Elbow and Silhouette methods and Gap statistic.

Figure S4. Unsupervised $\mathbf{k}$-means and pam clustering defines distinct metabolic subtypes of $B C$ tumors from dataset 2. (A) k-means and (B) pam of dataset 2 identified metabolomics clusters using $\mathrm{k}=3$. 
Figure S5. Identification of differential metabolites that define distinct metabolomic signatures of BC tumors from dataset 2. SAM identified differentially represented metabolites in the clusters from dataset 2. (A) False Discovery Rate (FDR) vs threshold value (Delta) plots for $\mathrm{k}=3$ analysis using $\mathrm{k}$ means, $\mathrm{FDR}=0$ at Delta=5.3 and 135 significant metabolites among these three clusters. (B) Heatmap of highly differentiated metabolites for 135 metabolites. Columns are samples and rows are metabolites. "Cluster" corresponds to metabolomic clusters.

Figure S6. Correlation between receptor and metabolic subtype. Statistical association between k-means clusters of dataset 2 and patients' molecular subtypes ( $p$-value $=0.0074$, Chi square test) derived from the Pearson's residual for cluster 3 , which contributed the most to luminal A subtype.

Figure S7. Identification of differential metabolites that define distinct metabolomic signatures of BC tumors. Same as Figures $3 \mathrm{C}$ and $3 \mathrm{D}$ with sample clustering using the hierarchical method.

Table S1. Differential metabolites in BC tumors. List of 90 metabolites identified by SAM in k-means clustered dataset 1 with $\mathrm{k}=4$. 


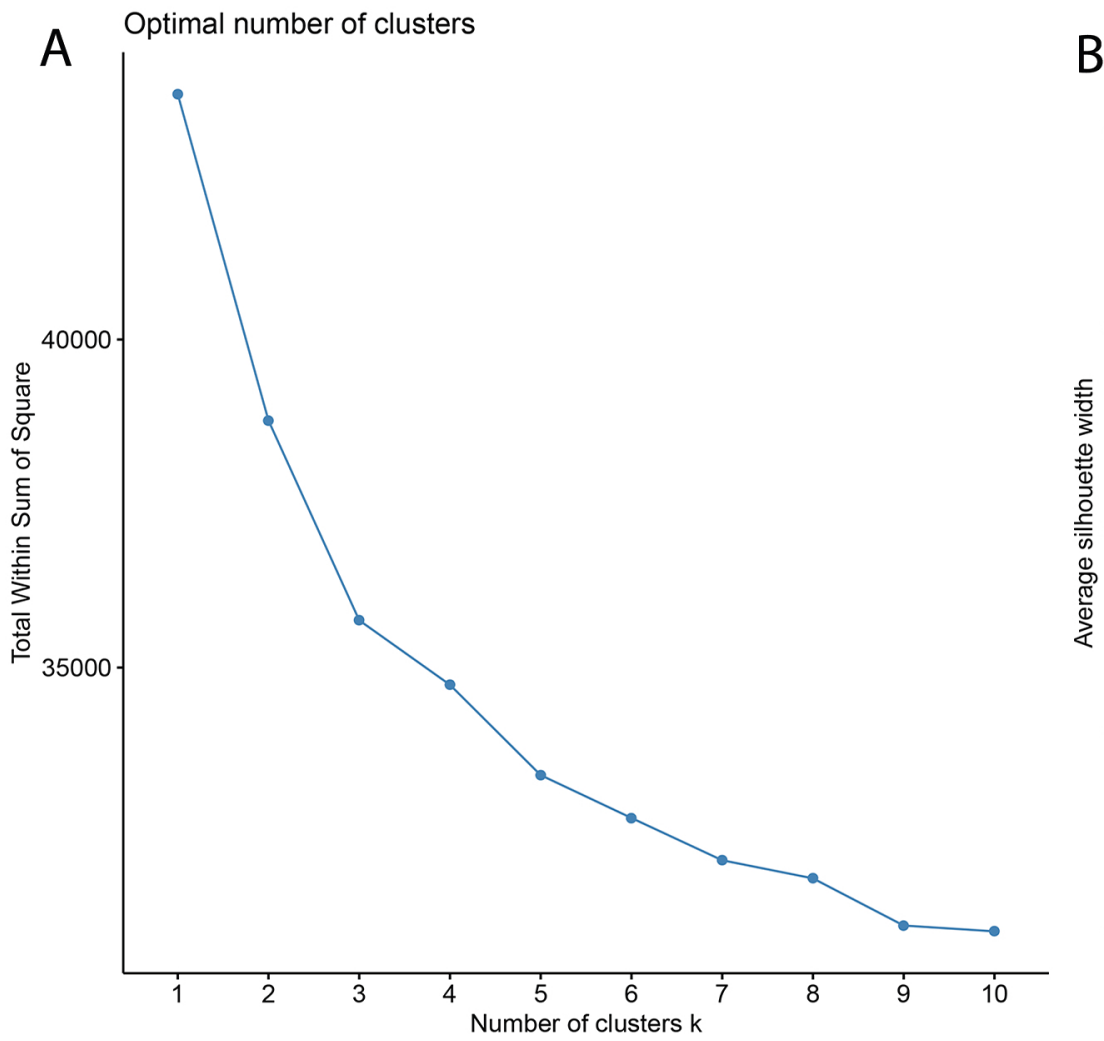

\section{R Optimal number of clusters}

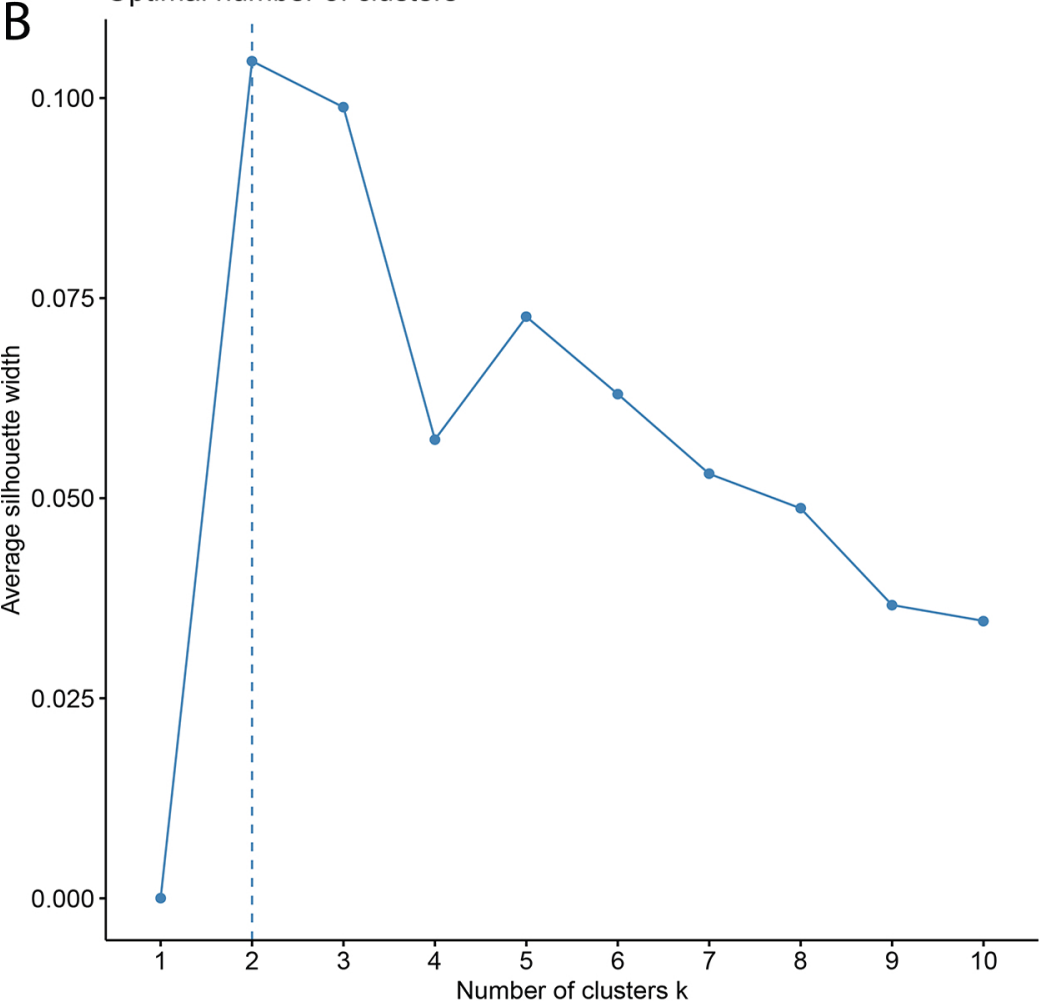

C Optimal number of clusters

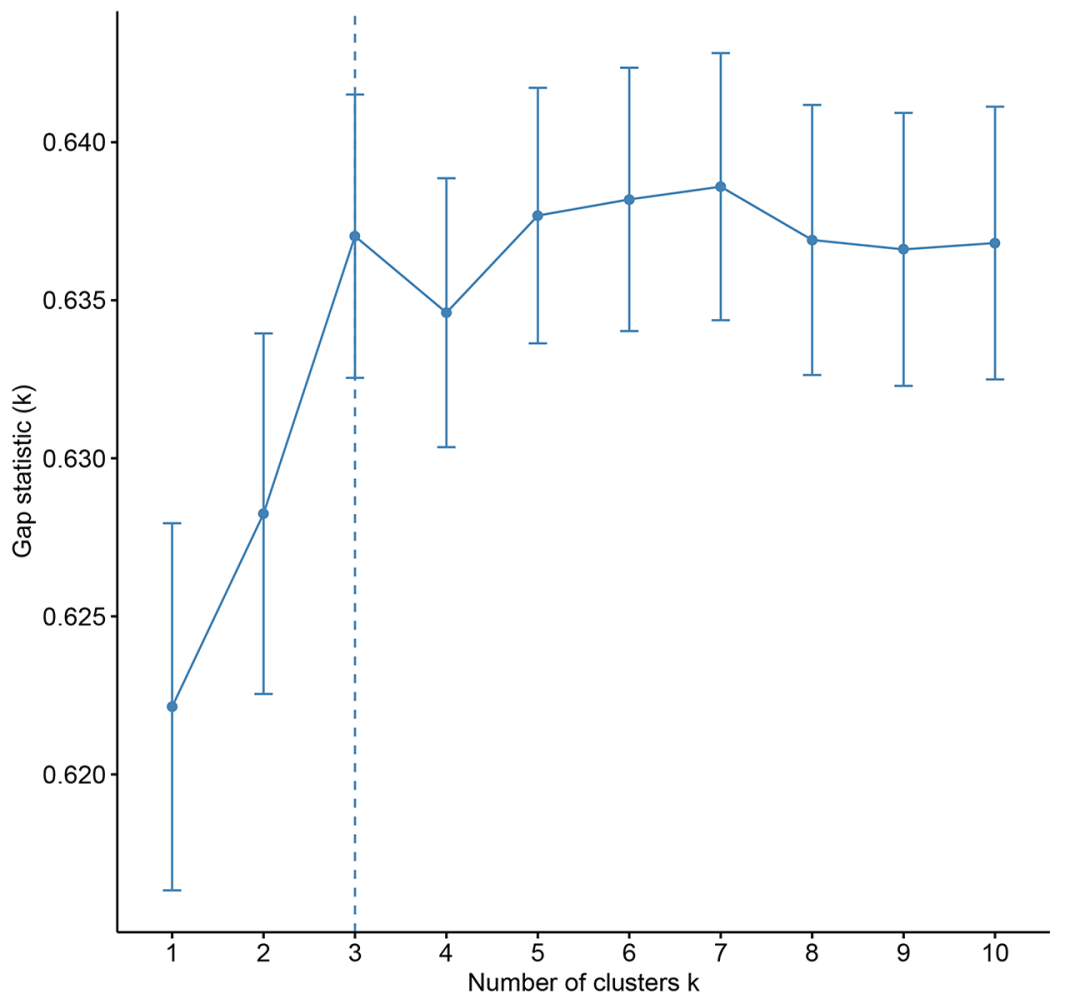

Figure S1: Analysis to find optimal k value for k-means clustering of dataset 1 by Elbow, Silhouette methods and Gap statistic.

S-3 


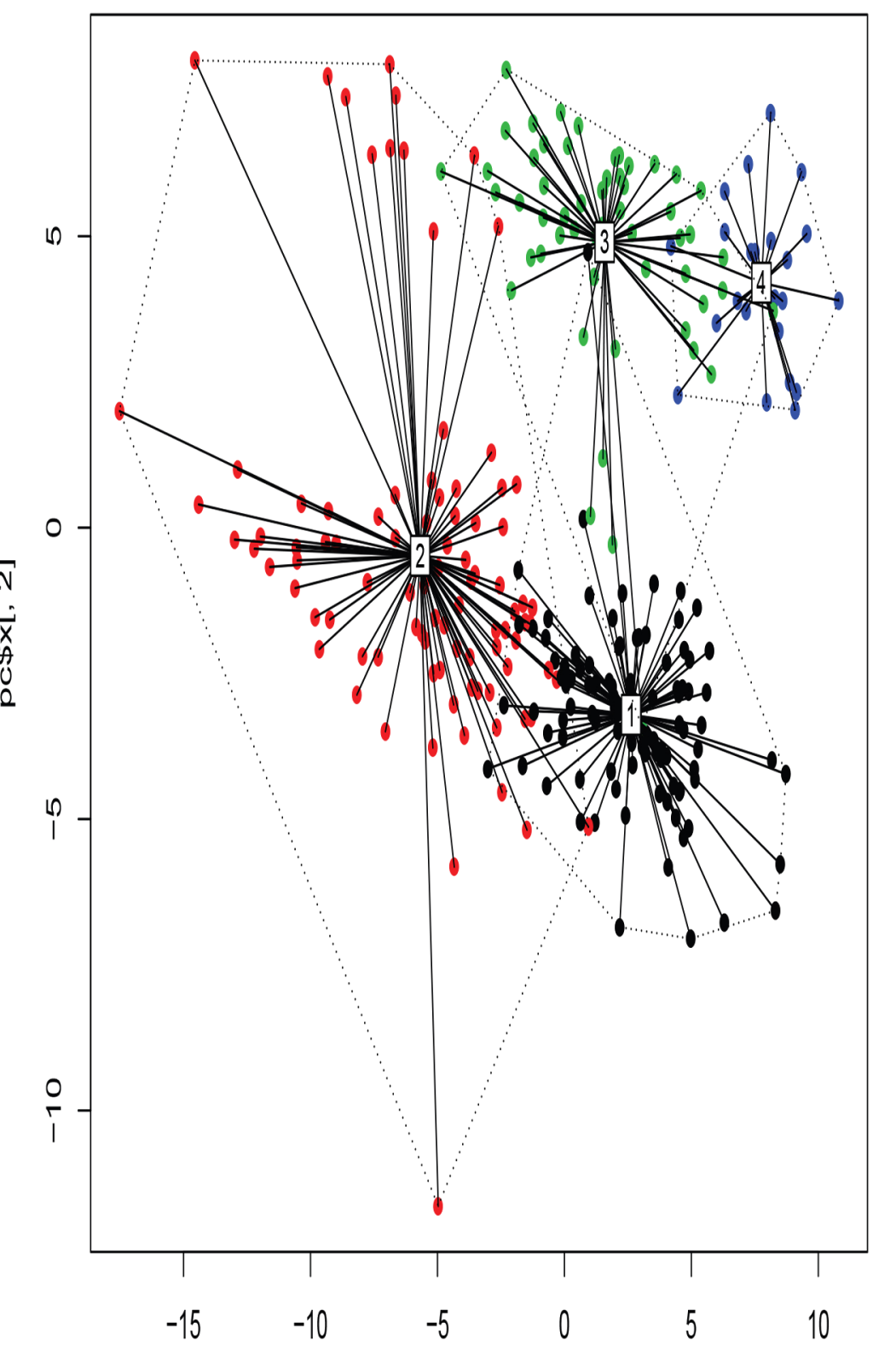

$\operatorname{pCSx[}, 1]$

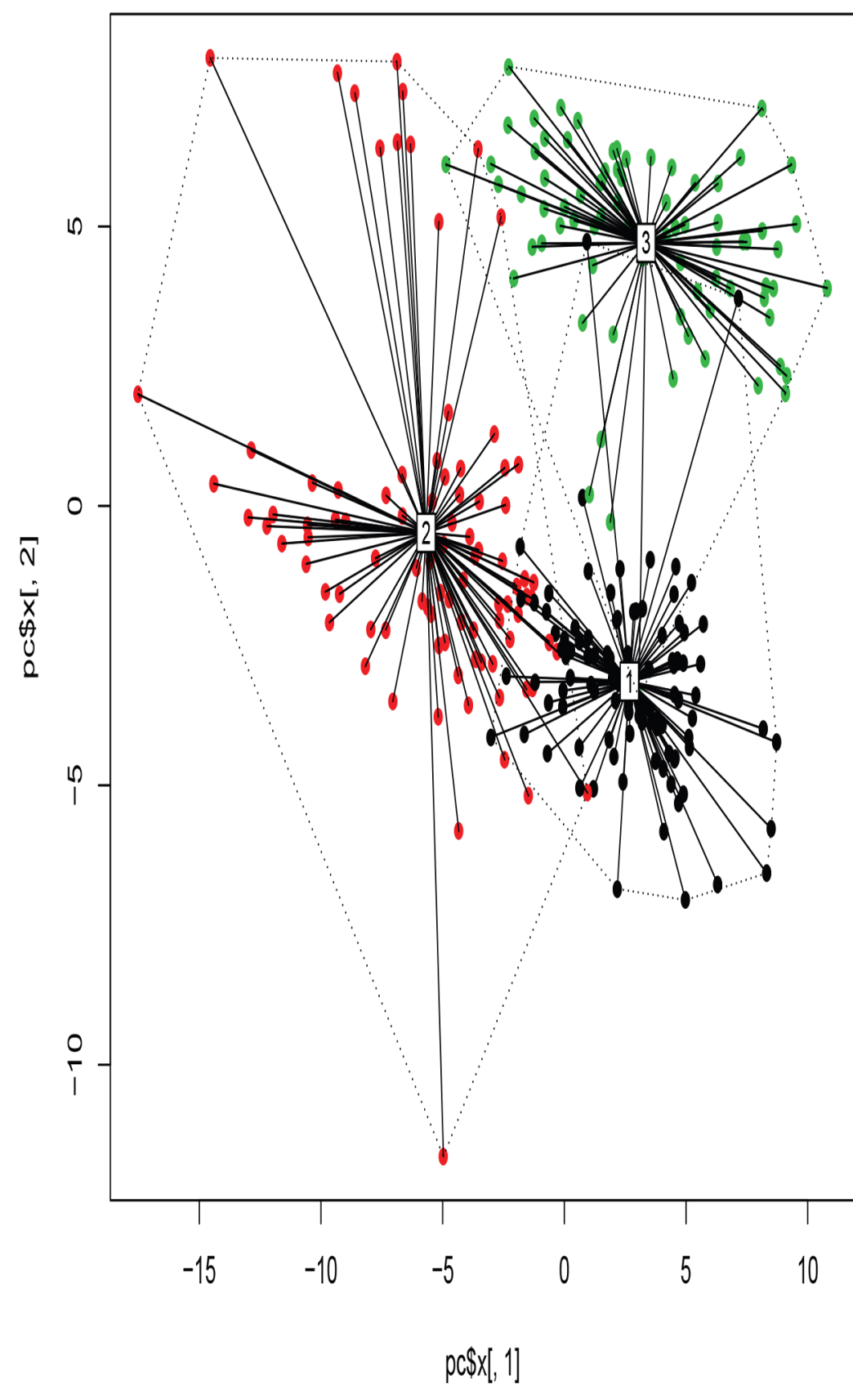

Figure S2: pam (partition around mediods) of dataset 1 identified metabolomics clusters using both, (A) $k=4$ (current number of receptor subtypes) and (B) $k=3$ (ideal $k$ value by Elbow method and Gap statistic). 

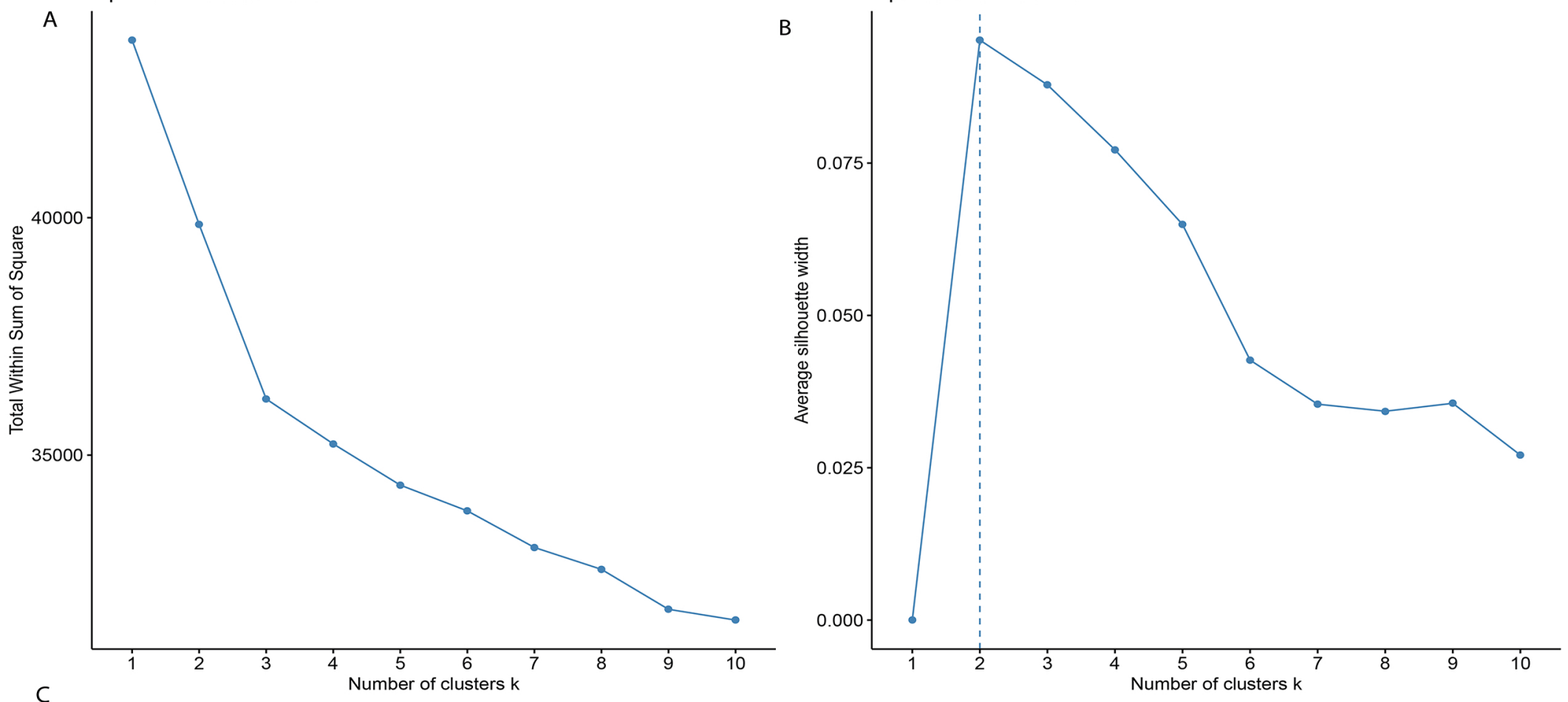

Optimal number of clusters

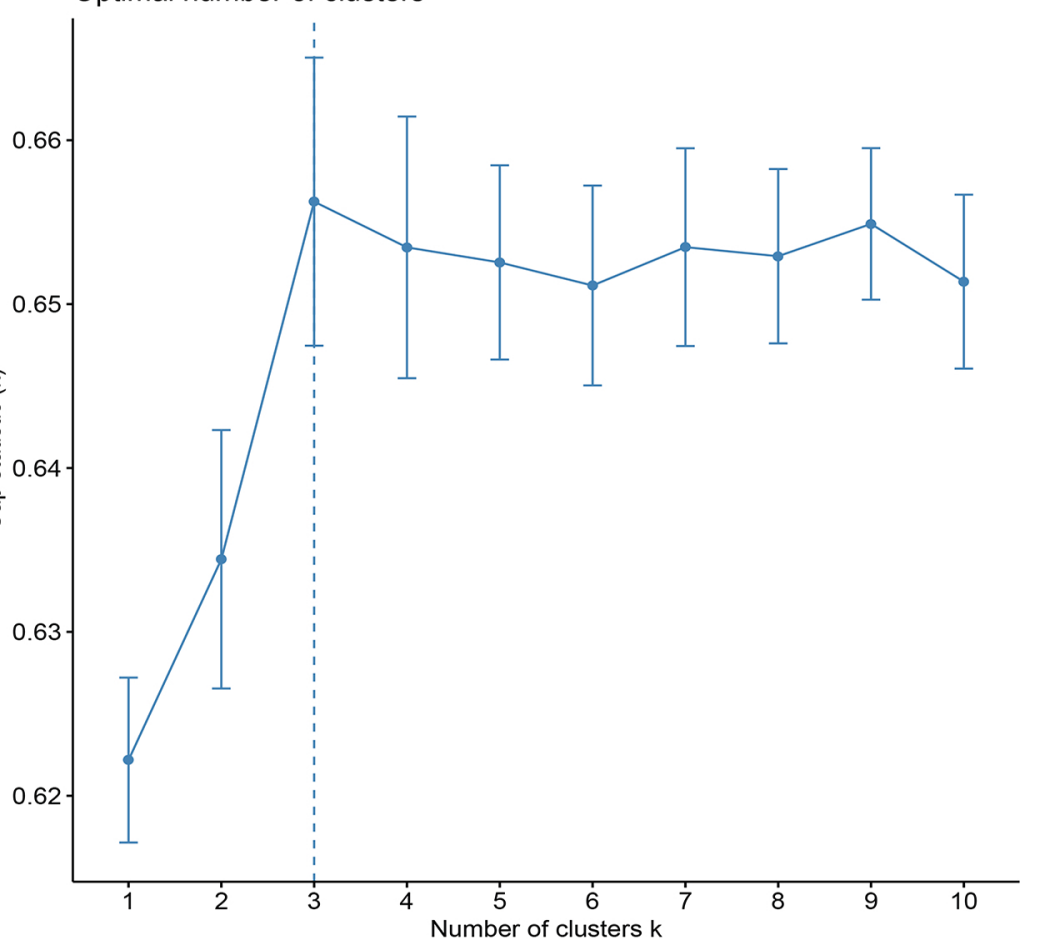

Figure S3: Analysis to find optimal k value for pam clustering of dataset 1 by Elbow and Silhouette methods and Gap statistic.

\section{S-5}


A

$\mathrm{K}$-mean clusters (Data2, $\mathrm{k}=3$ )

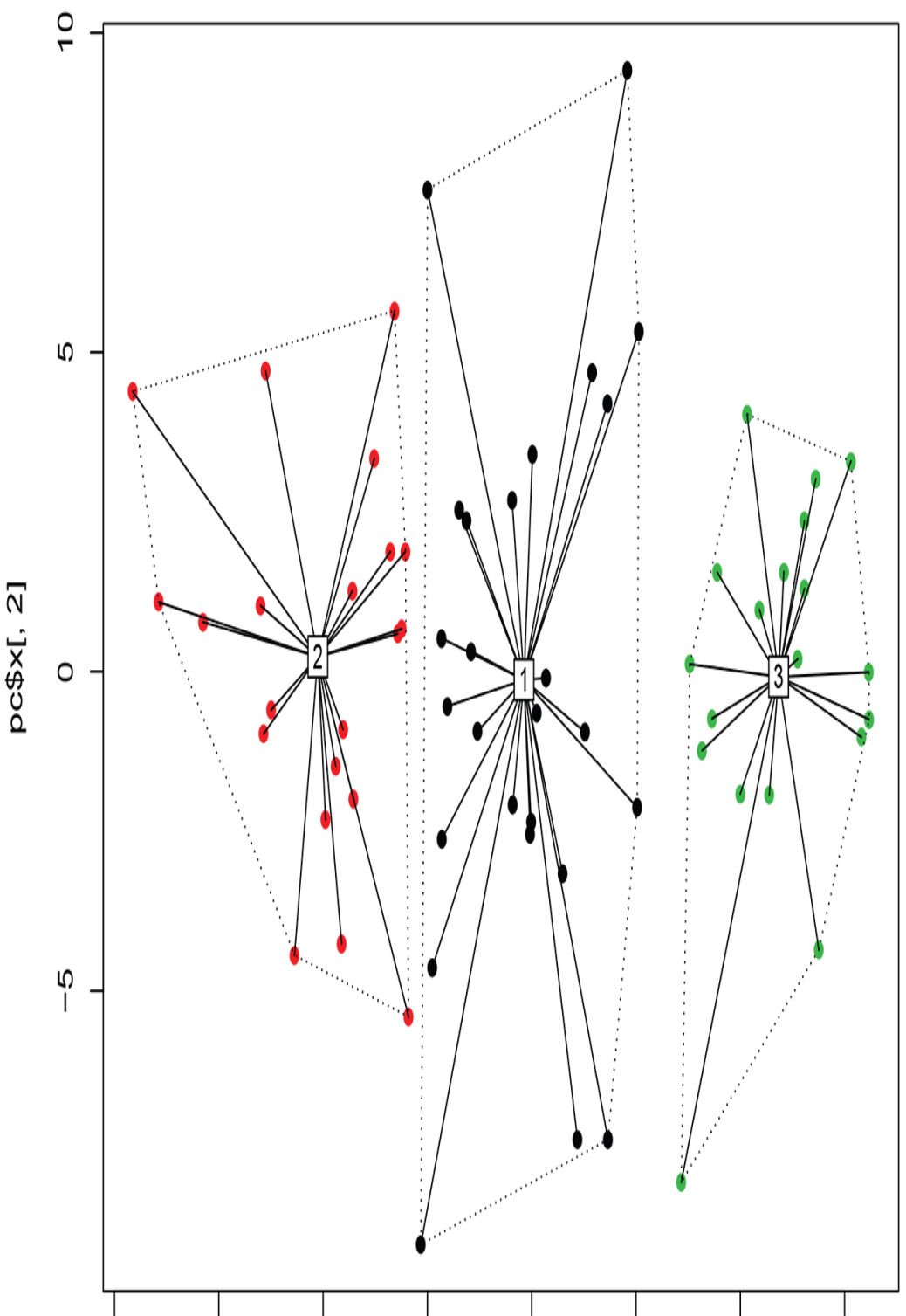

$\begin{array}{llllllll}-20 & -15 & -10 & -5 & 0 & 5 & 10 & 15\end{array}$

pcSX[, 1]

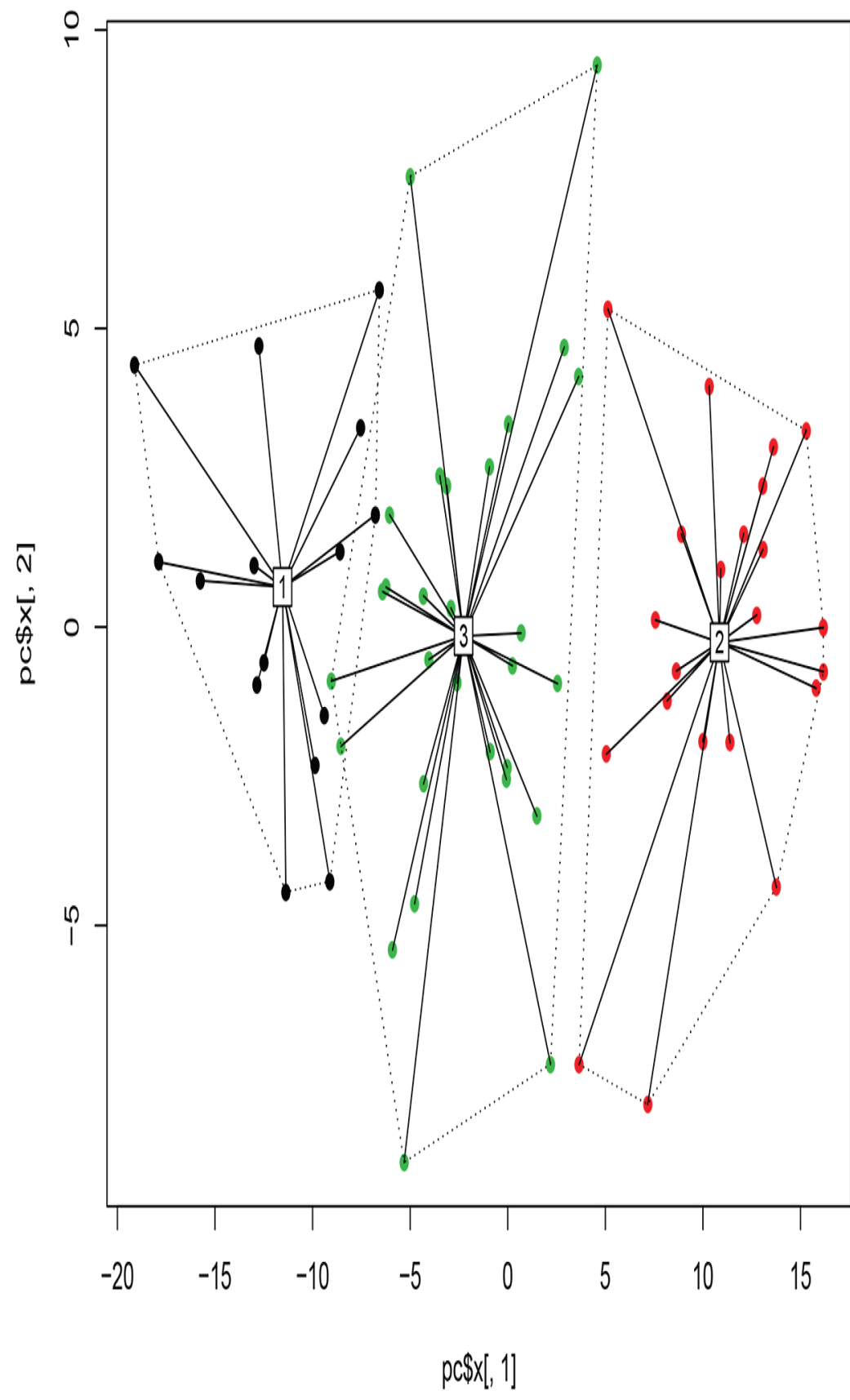

Figure S4: A) $k$-means and B) pam of dataset 2 identified metabolomics clusters using $k=3$. 

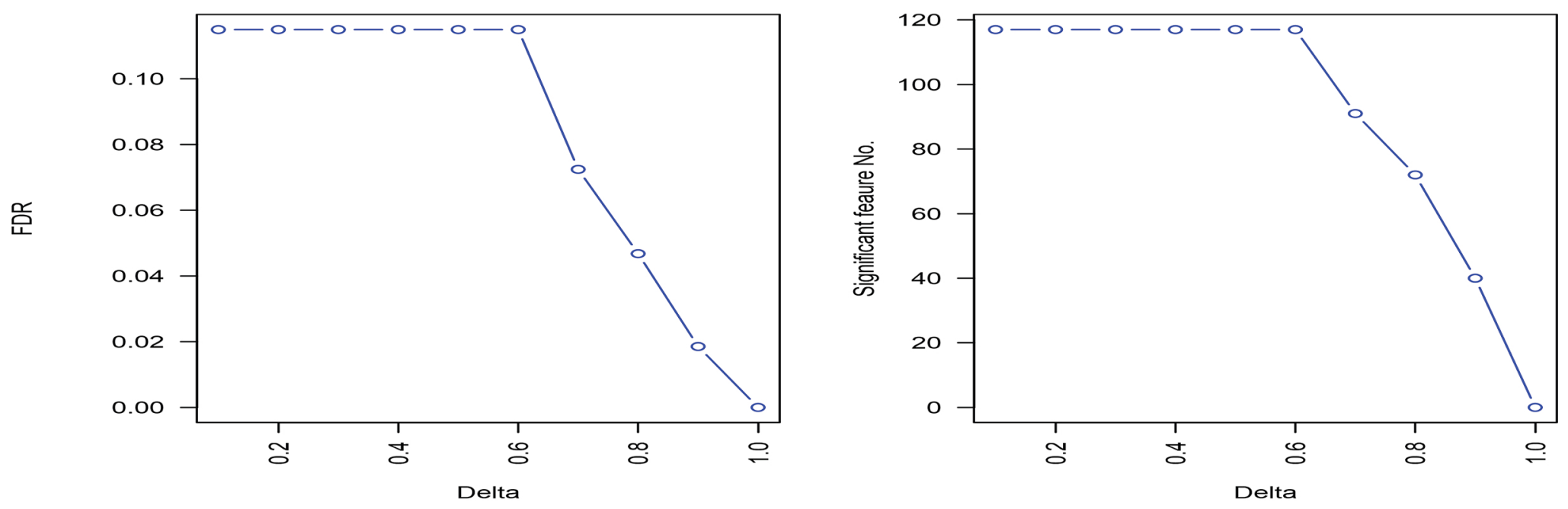

B

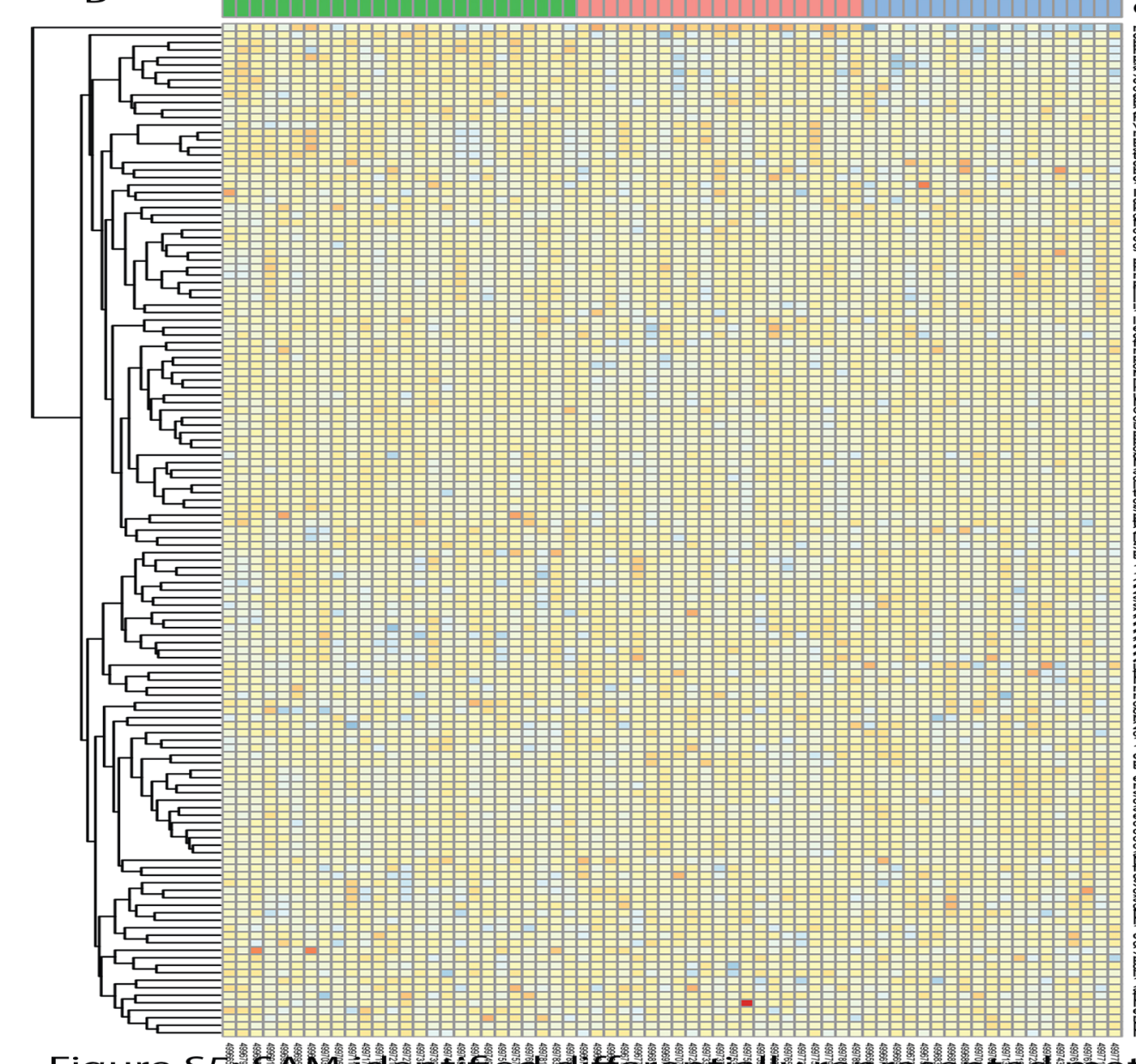

cluster

methionine sulfoxide

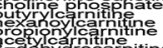

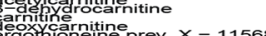

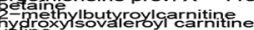

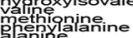

tring

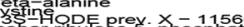

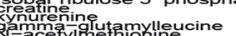

ofscolonate $181 \mathrm{nt}$

banimgtadeconoal

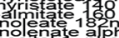

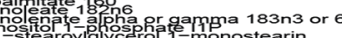

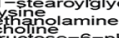

醇

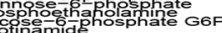

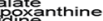

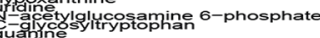

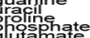

roc-actillysine

政

Natinghan

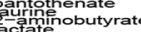

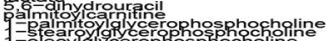

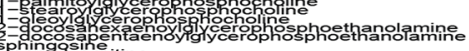

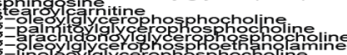

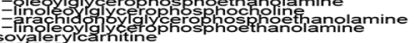

uncostetýlucosamine

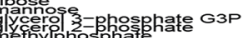

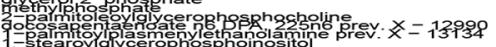

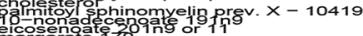

tateastive

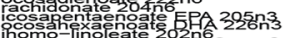

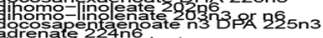

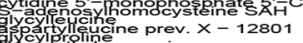

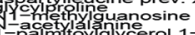

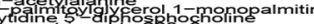

s.t.

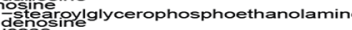

Hocosictilserine

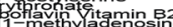

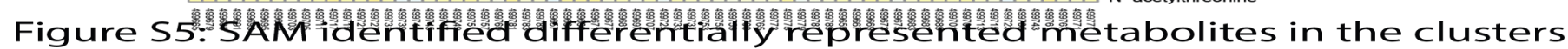
from dataset 2. (A) False Discovery Rate (FDR) vs threshold value (Delta) plots for $\mathrm{k}=3$ analysis using $\mathrm{kmean}, \mathrm{FDR}=\mathrm{O}$ at Delta $=5.3$ and 135 significant metabolites among these three clusters. (B) Heatmap of highly differentiated metabolites for 135 metabolites. Columns are samples and rows are metabolites. "Cluster" corresponds to metabolomic clusters. 


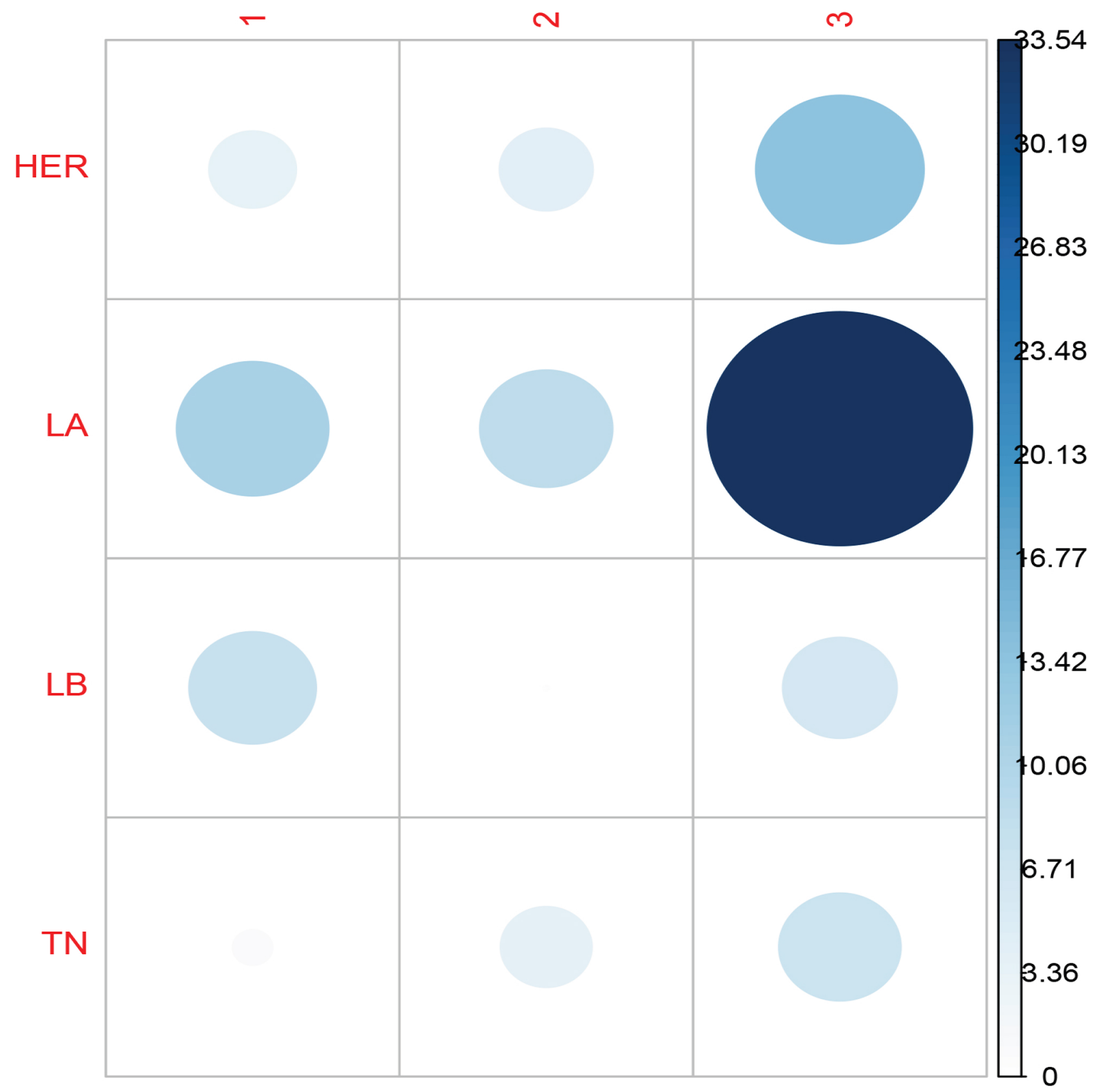

Figure S6: Statistical association between k-means clusters of dataset 2 and patients' molecular subtypes ( $p$-value $=0.0074$, Chi square test) derived from the Pearson's residual for cluster 3, which contributed the most to luminal A subtype.

S-8 


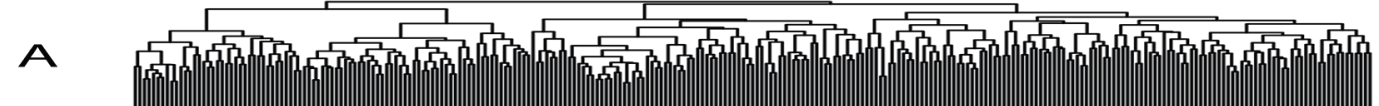
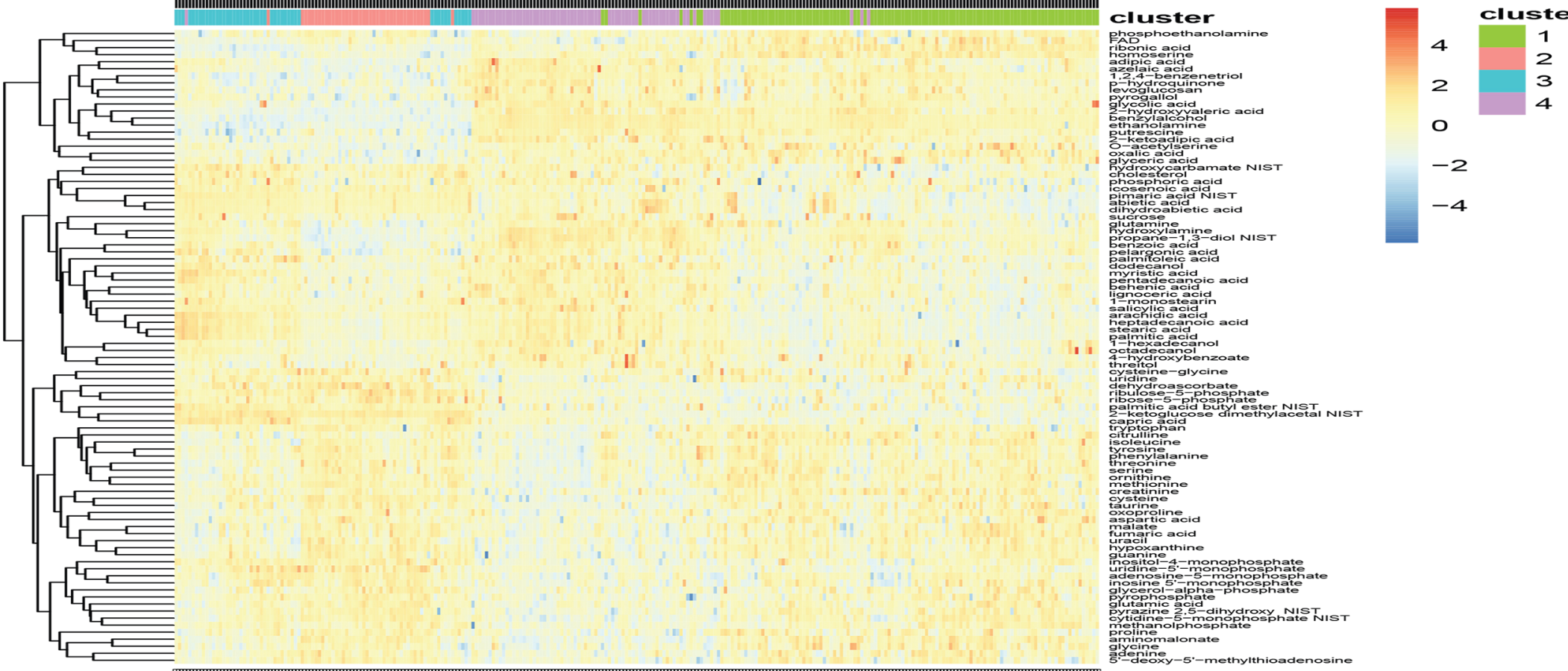

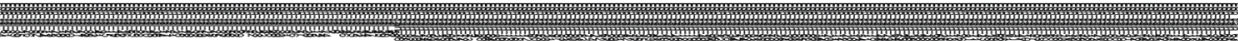

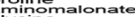

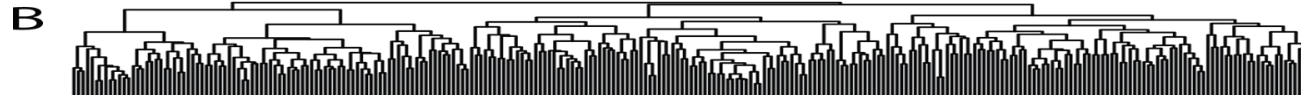

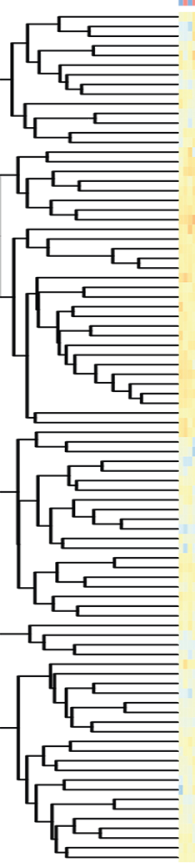

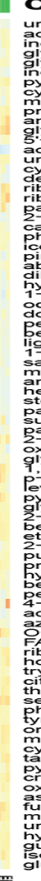

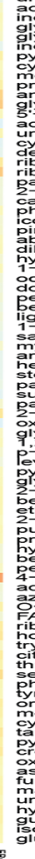

$r$

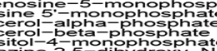

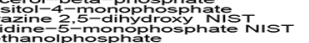

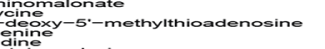

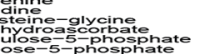

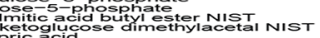

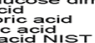

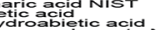

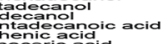

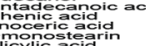

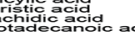

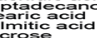

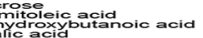

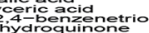

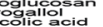

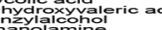

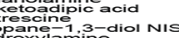

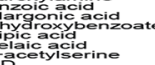

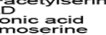

unilinio

Enyialanine

s.t.
strionine
rrinh

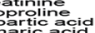

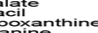

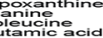

Figure S7: same as Figure $3 \subset$ and $D$ with clustering the samples using hierarchical method. 
Table S1: List of 90 metabolites identified by SAM in $\mathrm{k}$-means clustered dataset 1 with $\mathrm{k}=4$.

\begin{tabular}{|c|c|c|c|c|c|c|}
\hline & Name & HMDB & PubChem & ChEBI & KEGG & METLIN \\
\hline 1 & benzylalco & HMDBOOO & 244 & 17987 & 000556 & NA \\
\hline 2 & 2-ketogluc & NA & NA & NA & NA & NA \\
\hline 3 & ethanolam & HMDBOOO & 700 & 16000 & CO0189 & 3207 \\
\hline 4 & ribonic acid & HMDBOOO & 5460677 & 21077 & 001685 & 3290 \\
\hline 5 & 2-hydroxyy & HMDBOOO & 98009 & 60647 & & NA \\
\hline 6 & $1,2,4$-benz & METPAO3 & NA & NA & C02814 & NA \\
\hline 7 & capric acid & HMDBOOO & 2969 & 30813 & C01571 & 336 \\
\hline 8 & hydroxylan & HMDBOOO & 787 & 15429 & C00192 & 6894 \\
\hline 9 & propane-1 & NA & NA & NA & NA & NA \\
\hline 10 & ribulose-5- & NA & NA & NA & NA & NA \\
\hline 11 & stearic acid & HMDBOOO & 5281 & 28842 & C01530 & 189 \\
\hline 12 & putrescine & HMDBOOO & 1045 & 17148 & C00134 & 3226 \\
\hline 13 & salicylic ac & HMDBOOO & 338 & 16914 & 000805 & 616 \\
\hline 14 & homoserin & HMDBOOO & 12647 & 15699 & 000263 & 5687 \\
\hline 15 & arachidic a & HMDBOOO & 10467 & 28822 & C06425 & 401 \\
\hline 16 & aminomald & HMDBOOO & 100714 & 17475 & 000872 & NA \\
\hline 17 & glycolic aci & HMDBOOO & 757 & 17497 & C00160 & 3219 \\
\hline 18 & heptadeca & HMDBOOO & 10465 & 32365 & & 6578 \\
\hline 19 & 2-ketoadip & HMDBOOO & 71 & 15753 & 000322 & 5234 \\
\hline 20 & palmitic ac & HMDBOOO & 985 & 15756 & 000249 & 187 \\
\hline 21 & cysteine & HMDBOOO & 5862 & 17561 & 000097 & 5556 \\
\hline 22 & dehydroas & HMDBOOO & 210328 & 17242 & 000425 & 342 \\
\hline 23 & adipic acid & HMDBOOO & 196 & 30832 & C06104 & 115 \\
\hline 24 & myristic ac & HMDBOOO & 11005 & 28875 & C06424 & 196 \\
\hline 25 & uridine-5'-r & HMDBOOO & 6030 & 16695 & 000105 & NA \\
\hline 26 & oxalic acid & HMDBOOOA & 971 & 16995 & C00209 & 113 \\
\hline 27 & benzoic ac & HMDBOOO & 243 & 30746 & Co0180 & 1297 \\
\hline 28 & pimaric aci & NA & NA & NA & NA & NA \\
\hline 29 & hypoxanth & HMDBOOO & 790 & 17368 & C00262 & 83 \\
\hline 30 & ornithine & HMDBOOO & 6262 & 15729 & 000077 & 27 \\
\hline 31 & dodecanol & HMDBOO1 & 8193 & 28878 & 002277 & NA \\
\hline 32 & cytidine-5-1 & NA & NA & NA & NA & NA \\
\hline 33 & hydroxycai & NA & NA & NA & NA & NA \\
\hline 34 & inositol-4-n & NA & NA & NA & NA & NA \\
\hline 35 & methionine & HMDBOOO & 6137 & 16643 & C00073 & 5664 \\
\hline 36 & isoleucine & HMDBOOO & 6306 & 17191 & 000407 & 5193 \\
\hline 37 & azelaic aci & HMDBOOO & 2266 & 48131 & C08261 & 5750 \\
\hline 38 & threonine & HMDBOOO & 6288 & 16857 & Co0188 & 32 \\
\hline 39 & p-hydroqui & HMDBOOOA & 785 & 17594 & 000530 & 6681 \\
\hline 40 & glyceric ac & HMDBOOO & 439194 & 32398 & C00258 & 280 \\
\hline 41 & ribose-5-pl & HMDBOOO & 439167 & 52742 & CO0117 & 6315 \\
\hline 42 & 1-monoste & HMDBO03 & 24699 & 526874 & & NA \\
\hline 43 & pentadeca & HMDBOOO & 13849 & NA & C16537 & 5789 \\
\hline 44 & guanine & HMDBOOO & 764 & 16235 & 000242 & 315 \\
\hline 45 & palmitic ac & NA & NA & NA & NA & NA \\
\hline 46 & glutamic a & HMDBOOO & 33032 & 16015 & 000025 & 5174 \\
\hline 47 & taurine & HMDBOOO & 1123 & 15891 & COO245 & 31 \\
\hline 48 & behenic ad & HMDBOOO & 8215 & 28941 & C08281 & 260 \\
\hline
\end{tabular}




\begin{tabular}{|c|c|c|c|c|c|c|}
\hline 49 & FAD & HMDBOOO & 643975 & 16238 & Co0016 & 6106 \\
\hline 50 & levoglucos & HMDBOOO & 2724705 & 30997 & & 5613 \\
\hline 51 & \begin{tabular}{|l|} 
lignoceric \\
\end{tabular} & HMDBOOO & 11197 & 28866 & C08320 & 6427 \\
\hline 52 & O-acetylse & HMDBOOO & 99478 & 17981 & C00979 & 3270 \\
\hline 53 & pyrogallol & HMDB001 & 1057 & 16164 & C01108 & NA \\
\hline 54 & pyrazine 2 & $\mathrm{NA}$ & NA & NA & NA & NA \\
\hline 55 & abietic aci & $\mathrm{NA}$ & $\mathrm{NA}$ & NA & NA & NA \\
\hline 56 & dihydroabi & NA & NA & NA & NA & NA \\
\hline 57 & serine & HMDBOOO & 5951 & 17115 & C00065 & 5203 \\
\hline 58 & palmitoleic & HMDBOOO & 445638 & 28716 & C08362 & 188 \\
\hline 59 & glycine & HMDBOOO & 750 & 15428 & C00037 & 20 \\
\hline 60 & glutamine & HMDBOOO & 5961 & 18050 & C00064 & 5614 \\
\hline 61 & pyrophosp & HMDBOOO & 644102 & 18361 & C00013 & 3306 \\
\hline 62 & phosphori & HMDBOOO & 1004 & 26078 & C00009 & NA \\
\hline 63 & 4-hydroxyk & HMDBOOO & 135 & 30763 & C00156 & 5486 \\
\hline 64 & creatinine & HMDBOOO & 588 & 16737 & C00791 & 8 \\
\hline 65 & pelargonic & HMDBOOO & 8158 & 29019 & C01601 & 5810 \\
\hline 66 & malate & HMDBOOO & 222656 & 30797 & C00149 & NA \\
\hline 67 & 1-hexadec & HMDBOOO & 2682 & 16125 & C00823 & 6927 \\
\hline 68 & adenosine & $\mathrm{NA}$ & NA & NA & NA & NA \\
\hline 69 & oxoproline & HMDBOOO & 7405 & 18183 & C01879 & 3251 \\
\hline 70 & fumaric ac & HMDBOOO & 444972 & 18012 & C00122 & 3242 \\
\hline 71 & cholestero & HMDBOOO & 11025495 & 1307929 & C00187 & 163 \\
\hline 72 & sucrose & HMDBOOO & 5988 & 17992 & C00089 & 137 \\
\hline 73 & tyrosine & HMDBOOO & 6057 & 17895 & C00082 & 34 \\
\hline 74 & cysteine-g & $\mathrm{NA}$ & NA & $\mathrm{NA}$ & NA & NA \\
\hline 75 & proline & HMDBOOO & 145742 & 17203 & Co0148 & 29 \\
\hline 76 & phenylalar & HMDBOOO & 6140 & 17295 & C00079 & 28 \\
\hline 77 & uridine & HMDBOOO & 6029 & 16704 & C00299 & 90 \\
\hline 78 & uracil & HMDBOOO & 1174 & 17568 & C00106 & 258 \\
\hline 79 & citrulline & HMDBOOO & 9750 & 16349 & C00327 & 16 \\
\hline 80 & adenine & HMDBOOO & 190 & 16708 & C00147 & 85 \\
\hline 81 & aspartic ac & HMDBOOO & 5960 & 17053 & C00049 & 5206 \\
\hline 82 & 5'-deoxy-5 & HMDBOOO & 439176 & 17509 & C00170 & 3425 \\
\hline 83 & phosphoet & HMDBOOO & 1015 & 17553 & C00346 & 5233 \\
\hline 84 & inosine 5'- & HMDBOOO & 8582 & 17202 & C00130 & 5196 \\
\hline 85 & methanolp & $\mathrm{NA}$ & NA & $\mathrm{NA}$ & NA & NA \\
\hline 86 & icosenoic & $\mathrm{NA}$ & NA & $\mathrm{NA}$ & NA & NA \\
\hline 87 & octadecan & HMDBOOO & 8221 & 32154 & D01924 & 6640 \\
\hline 88 & tryptophan & HMDBOOO & 6305 & 16828 & C00078 & 5879 \\
\hline 89 & threitol & HMDBOOO & 169019 & 48300 & C16884 & NA \\
\hline 90 & glycerol-al & $\mathrm{NA}$ & NA & $\mathrm{NA}$ & NA & NA \\
\hline
\end{tabular}

